\title{
VALIDAÇÃO DOS TESTES DE EWING PARA AVALIAÇÃO DE DISFUNÇÃO AUTONÔMICA
}

\author{
CARLOS ALBERTO DE ALMEIDA BOER*, ALTAIR JACOB MOCELIN**, TIEMI MATSUO***
}

\begin{abstract}
RESUMO - A necessidade de padronização dos testes para avaliar a disfunção autonômica tornou-se evidente a partir dos resultados contraditórios observados nas últimas duas décadas. Os testes propostos por Ewing e col., com este objetivo, têm ampliado sua aceitação; avaliam os reflexos cardiovasculares, mas podem refletir dano em qualquer outro ponto do sistema nervoso autônomo. Propusemos validá-los em nosso meio, aplicando-os em 30 indivíduos sadios e 23 diabéticos, representando os parâmetros de normalidade e anormalidade, respectivamente. Os 5 testes demonstraram alto grau de sensibilidade e especificidade, são reprodutíveis, realizados com um mínimo de material simples, servindo à sua proposta.
\end{abstract}

PALAVRAS-CHAVE: disfunção autonômica, sistema nervoso autônomo, diabetes, testes de Ewing, testes para disfunção autonômica.

\section{Ewing's tests validation for autonomic dysfunction}

ABSTRACT - The medical literature on the evaluation of autonomic dysfunction accumulated discordant results in the past decades, accentuating the need of validating the proposed tests. The Ewing's tests for studying autonomic abnormalities received increasing acceptance, as they recognize not only cardiovascular reflexes damage but lesions in any other segments of the autonomic nervous system. We proposed to validate them in our population by analysing their results on 30 normal individuals and 23 long-standing diabetics. Our results show that the five tests are highly sensitive and specific, reproducible, use simple and available office material, and fulfil their purpose.

KEY WORDS: autonomic dysfunction, autonomic nervous system, diabetes, Ewing's tests, autonomic dysfunction testing.

Desde o surgimento dos primeiros testes para avaliação do sistema nervoso autônomo, na década de 60, observa-se aumento do interesse em identificar e quantificar as disfunções deste sistema. Possivelmente, o maior interesse despertado pelas degenerações neuronais primárias envolvendo as vias autonômicas, recentemente classificadas ${ }^{4}$, e, também, a identificação da presença de disfunção autonômica em grande variedade de condições clínicas, produzindo nítido agravamento destas, como a correlação de sua presença com o prognóstico do enfarto agudo do miocárdio ${ }^{2}$, obrigam ao desenvolvimento de testes que informem a presença e o grau de disfunção. Os estudos iniciais foram pautados por resultados contraditórios, gerando controvérsias acerca da presença e grau de disfunção autonômica ${ }^{1,3,8-11}$, sendo logo apontada a falta de padronização dos testes, como a

Centro de Ciências da Saúde da Universidade Estadual de Lodrina: *Neurologista, Mestre em Medicina Interna; **Professor Adjunto, Departamento de Clínica Médica; ***Professora Adjunto, Departamento de Matemática Aplicada e Estatística. Aceite: 16-janeiro-1998.

Este estudo faz parte da Tese de Mestrado (CAAB) "Avaliação da disfunção autonômica em pacientes portadores de insuficiência renal crônica" aprovado no Curso de Pós-Graduação em Medicina Interna da Universidade Estadual de Londrina PR e realizado no Laboratório de Pós-Graduação em Medicina Interna.

Dr. Carlos Alberto de Almeida Boer - Universidade Estadual de Londrina, Centro de Ciências da Saúde - Rua Robert Koch 60 - 86030-440 Lodrina PR - Brasil. Fax 0433211824. 
responsável. No início da última década, Ewing e col. propuseram tal padronização ${ }^{5-7}$, utilizando testes que avaliam os reflexos cardiovasculares mas que podem refletir dano em qualquer outro ponto do sistema nervoso autônomo, tanto da via simpática quanto da parassimpática. Após serem recomendados pela "Conferência de San Antonio" sobre neuropatia diabética, em 1988 ${ }^{12}$, ganharam maior aceitação e uso frequente em diversos ensaios clínicos.

Propusemos validá-los em nosso meio, aplicando-os em indivíduos sadios e em pacientes com probabilidade de apresentarem disfunção do sistema nervoso autônomo.

\section{MATERIAL E MÉTODOS}

Foram selecionados dois grupos de indivíduos voluntários: um representando os parâmetros de normalidade, composto por 30 indivíduos sadios, com idade variando entre 15 e 57 anos (média 34,9 anos), sendo 21 do sexo feminino e 9 do masculino; o outro representando os parâmetros de anormalidade, composto por 23 pacientes de uma população de diabéticos há mais de 10 anos usando hipoglicemiantes, com idade variando entre 32 e 77 anos (média 52,5 anos), sendo 6 do sexo feminino e 17 do masculino, 11 eram normotensos e 12 hipertensos em uso de medicação. Todos os testes foram aplicados pelo mesmo médico, que necessitou de 30 minutos para avaliar cada indivíduo. O material utilizado constou de: manômetro de mercúrio (GERAR), eletrocardiógrafo (Funbec - ECG-40), dinamômetro de mão (METER), estetoscópio (Littmann); para o teste de esforço da manobra de Valsalva, foi adaptado um bocal descartável a uma traquéia, destas usadas em prolongamentos de ventiladores mecânicos, e este conjunto ligava-se ao manômetro de mercúrio.

Num total de 5 testes, 3 avaliam a frequência cardíaca (variação da frequência cardíaca à respiração profunda, manobra de Valsalva e a razão 30/15) refletindo predominantemente a função parassimpática e 2 a pressão arterial (variação da pressão arterial ao se levantar e preensão) aferindo principalmente a função simpática.

O estudo da variação da frequência cardíaca, durante a respiração profunda é feito com o paciente sentado e calmo, solicitando-se que faça 6 respirações profundas em 1 minuto, inspirando durante 5 segundos e expirando por outros 5 segundos. Durante este período é realizado eletrocardiograma, marcando-se no traçado o início de cada inspiração e expiração. O menor intervalo R-R da inspiração e o maior da expiração, em cada ciclo, são convertidos em batimentos por minutos (bpm). O resultado é expresso como a média da diferença da frequência cardíaca máxima e a mínima dos 6 ciclos, dentro dos limites: normal, $\geq 15$ bpm; limítrofe, 11 a 14 bpm; anormal, $\leq 10 \mathrm{bpm}$.

A resposta da frequência cardíaca à manobra de Valsalva é avaliada com o indivíduo sentado, soprando em um bico conectado a manômetro de mercúrio com esforço suficiente para manter a pressão expiratória em 40 $\mathrm{mm} \mathrm{Hg}$ e por 15 segundos, enquanto se registra continuamente o eletrocardiograma, que se continua por outros 15 segundos após a liberação do esforço. A manobra é realizada 3 vezes, com intervalo de 1 minuto. O resultado é expresso em taxa de Valsalva, que é a razão entre as durações do maior intervalo R-R após a manobra (bradicardia) e do menor intervalo R-R durante o esforço (taquicardia do esforço). A medida é feita com régua milimetrada, no traçado eletrocardiográfico. A média das 3 razões é aceita como valor final, dentro dos seguintes parâmetros: normal, $\geq 1,21 \mathrm{~mm} / \mathrm{seg}$; limítrofe, 1,11 a $1,21 \mathrm{~mm} / \mathrm{seg}$; anormal, $\leq 1,10 \mathrm{~mm} / \mathrm{seg}$.

O teste da razão 30/15 (resposta imediata da frequência cardíaca ao levantar) se inicia com o indivíduo deitado e com registro eletrocardiográfico contínuo. A seguir, ele é solicitado a levantar-se de uma só vez. O instante em que coloca o pé no chão é anotado no traçado eletrocardiográfico que continua registrando até o $31^{\circ}$ batimento. O encurtamento do intervalo R-R, entre o $15^{\circ}$ e $16^{\circ}$ batimentos, e o alongamento do intervalo do $30^{\circ}$ e o seguinte são medidos com régua. A taxa cardíaca é expressa pela razão 30/15, dentre dos limites: normal, $\geq$ 1,04 ; limítrofe, 1,01 a 1,03; anormal, $\leq 1,00$.

O teste para avaliar a resposta da pressão arterial ao se levantar é realizado medindo a pressão arterial com o indivíduo deitado e relaxado, por 3 a 5 minutos e, novamente, no primeiro minuto após se levantar. $\mathrm{O}$ cálculo é feito pela diferença das pressões sistólicas, deitado e a em pé. Este simples teste é anormal somente quando houver severo comprometimento simpático, e seus limites são os seguintes: normal, $\leq 10 \mathrm{mmHg}$; limítrofe, 11 a $29 \mathrm{mmHg}$; anormal, $\geq 30 \mathrm{mmHg}$.

A resposta da pressão arterial à preensão sustentada é avaliada com o paciente sentado e em repouso, a pressão arterial sendo medida 3 vezes. Determina-se o máximo de contração voluntária usando um dinamômetro de mão. Solicita-se que mantenha a preensão em $30 \%$ do valor da contração máxima, por tempo o quanto possível além de 5 minutos. A pressão arterial é medida em intervalos de 1 minuto, durante o tempo que durar a preensão. 
Tabela 1. Resultados dos testes em 30 indivíduos sadios.

\begin{tabular}{|c|c|c|c|c|c|c|}
\hline Caso & $\begin{array}{l}\text { Valsalva } \\
\mathrm{mm} / \mathrm{seg}\end{array}$ & $\begin{array}{c}\text { Respiração } \\
\text { profunda } \\
\text { bpm }\end{array}$ & $\begin{array}{c}\text { Razão } \\
\text { 30/15 } \\
\text { mm/seg }\end{array}$ & $\begin{array}{c}\text { Variação da } \\
\text { pressão } \\
\text { mm Hg }\end{array}$ & $\begin{array}{l}\text { Preensão } \\
\text { mm Hg }\end{array}$ & Escore \\
\hline 1 & 1,33 & 37 & 1,07 & 0 & 28 & 0 \\
\hline 2 & 1,67 & 26 & 1,12 & 0 & 29 & 0 \\
\hline 3 & 1,29 & 37 & 1,41 & 0 & 30 & 0 \\
\hline 4 & 1,48 & 32 & 1,17 & 3 & 25 & 0 \\
\hline 5 & 1,73 & 19 & 1,05 & 6 & 22 & 0 \\
\hline 6 & 1,36 & 25 & 1,06 & 0 & 15 & 1 \\
\hline 7 & 1,25 & 18 & 1,12 & 6 & 55 & 0 \\
\hline 8 & 2,27 & 27 & 1,15 & 10 & 55 & 0 \\
\hline 9 & 1,35 & 15 & 1,05 & 0 & 30 & 0 \\
\hline 10 & 1,46 & 21 & 1,05 & 6 & 40 & 0 \\
\hline 11 & 1,44 & 29 & 1,40 & 0 & 25 & 0 \\
\hline 12 & 1,84 & 15 & 1,18 & 0 & 29 & 0 \\
\hline 13 & 1,59 & 15 & 1,16 & 0 & 33 & 0 \\
\hline 14 & 1,77 & 12 & 1,06 & 0 & 25 & 1 \\
\hline 15 & 1,45 & 15 & 1,29 & 0 & 47 & 0 \\
\hline 16 & 1,16 & 16 & 1,11 & 0 & 35 & 1 \\
\hline 17 & 1,88 & 19 & 1,21 & 0 & 35 & 0 \\
\hline 18 & 2,16 & 25 & 1,02 & 0 & 20 & 1 \\
\hline 19 & 1,67 & 28 & 1,15 & 5 & 30 & 0 \\
\hline 20 & 1,24 & 27 & 1,31 & 0 & 20 & 0 \\
\hline 21 & 1,17 & 19 & 1,14 & 0 & 18 & 1 \\
\hline 22 & 1,22 & 23 & 1,30 & 0 & 35 & 0 \\
\hline 23 & 1,70 & 15 & 1,06 & 0 & 30 & 0 \\
\hline 24 & 1,24 & 32 & 1,14 & 0 & 25 & 0 \\
\hline 25 & 1,40 & 14 & 1,12 & 0 & 35 & 1 \\
\hline 26 & 1,71 & 19 & 1,11 & 8 & 28 & 0 \\
\hline 27 & 1,49 & 12 & 1,12 & 0 & 24 & 1 \\
\hline 28 & 1,37 & 28 & 1,05 & 2 & 39 & 0 \\
\hline 29 & 1,24 & 22 & 1,13 & 8 & 42 & 0 \\
\hline 30 & 1,42 & 21 & 1,05 & 9 & 34 & 0 \\
\hline Médias & 1,51 & 22,1 & 1,14 & 2,1 & 31,3 & 0,23 \\
\hline Desvio padrão & 0,28 & 7,1 & 0,10 & 3,3 & 9,7 & 0,43 \\
\hline
\end{tabular}

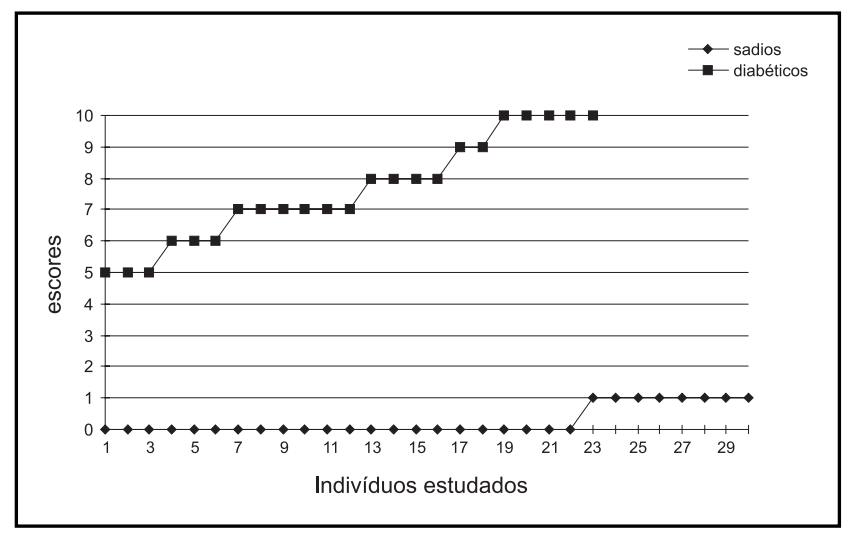

Fig 1. Gráfico dos resultados dos testes em escores propostos por Ewing. 
O resultado é expresso como a diferença entre a maior pressão diastólica, durante o esforço, e a média das 3 pressões diastólicas determinadas durante o repouso, tendo como limites: normal, $\geq 16 \mathrm{mmHg}$; limítrofe, 11 a 15 $\mathrm{mmHg}$; anormal, $\leq 10 \mathrm{mmHg}$.

Os dados foram submetidos a análise descritiva, utilizando-se as estatísticas média, desvio padrão, proporção, tabelas e gráficos. No cálculo da sensibilidade e especificidade, considerou-se o intervalo de confiança (IC) de $95 \%$. Todos os testes foram realizados ao nível de significância de $5 \%$.

Ewing e col..$^{5-7}$ propõem duas formas de interpretação dos resultados dos testes: classificação por grupos ou atribuição de escores. Optamos pela última, por permitir maior escalonamento da severidade. Nesta, para cada teste atribuiu-se o valor: 0 (zero) para o resultado normal; 1 , para o limítrofe; 2, para o anormal. Considerando tratar-se de 5 testes para cada indivíduo, a soma dos escores deverá situar-se entre 0 (zero) e 10, permitindo amplo escalonamento da severidade.

\section{RESULTADOS}

No grupo dos sadios (Tabela 1), 23 indivíduos apresentaram todos os testes normais e apenas $7(23,3 \%)$ apresentaram 1 dos testes com resultado limítrofe; portanto, para estes, o escore foi 1. Já, todos diabéticos (Fig 1) apresentaram resultados anormais, nenhum apresentando escore inferior a 5, e para 5 pacientes o escore foi máximo. A média dos escores dos hipertensos foi 7,66 e dos normotensos 7,54 (Tabela 2).

O teste da respiração profunda (Tabela 2) foi anormal em todos os diabéticos; na manobra de Valsalva apenas 1 caso foi limítrofe, sendo os demais (95,65\%) anormais. Para o teste da razão,

Tabela 2. Resultados dos testes em 23 pacientes diabéticos.

\begin{tabular}{|c|c|c|c|c|c|c|c|}
\hline Caso & $\begin{array}{l}\text { Valsalva } \\
\mathrm{mm} / \mathrm{seg}\end{array}$ & $\begin{array}{c}\text { Respiração } \\
\text { Profunda } \\
\text { bpm }\end{array}$ & $\begin{array}{c}\text { Razão } \\
30 / 15 \\
\mathrm{~mm} / \mathrm{seg}\end{array}$ & $\begin{array}{c}\text { Variação } \\
\text { da pressão } \\
\text { mm Hg }\end{array}$ & $\begin{array}{l}\text { Preensão } \\
\text { mm Hg }\end{array}$ & Escores & $\begin{array}{c}\text { Hipertensão } \\
\text { Arterial } \\
\text { (HA) }\end{array}$ \\
\hline 1 & 1,10 & 3 & 1,05 & 22 & 12 & 6 & HA \\
\hline 2 & 1,00 & 5 & 1,00 & 25 & 3 & 9 & - \\
\hline 3 & 1,00 & 3 & 1,00 & 5 & 15 & 7 & - \\
\hline 4 & 1,04 & 0 & 1,00 & 40 & 6 & 10 & HA \\
\hline 5 & 1,00 & 10 & 1,00 & 28 & 21 & 7 & - \\
\hline 6 & 1,02 & 1 & 1,00 & 50 & 25 & 8 & HA \\
\hline 7 & 1,01 & 6 & 1,00 & 37 & 16 & 8 & - \\
\hline 8 & 1,05 & 0 & 1,02 & 25 & 17 & 6 & - \\
\hline 9 & 1,00 & 0 & 1,00 & 20 & 22 & 7 & HA \\
\hline 10 & 1,00 & 0 & 1,00 & 77 & 6 & 10 & - \\
\hline 11 & 1,08 & 9 & 1,16 & 12 & 25 & 5 & HA \\
\hline 12 & 1,00 & 5 & 1,00 & 15 & 16 & 7 & HA \\
\hline 13 & 1,00 & 1 & 1,00 & 34 & 4 & 10 & HA \\
\hline 14 & 1,01 & 3 & 1,00 & 38 & 12 & 9 & HA \\
\hline 15 & 1,03 & 4 & 1,05 & 37 & 25 & 6 & HA \\
\hline 16 & 1,05 & 2 & 1,00 & 25 & 14 & 8 & - \\
\hline 17 & 1,00 & 0 & 1,00 & 40 & 4 & 10 & HA \\
\hline 18 & 1,00 & 5 & 1,02 & 10 & 7 & 7 & HA \\
\hline 19 & 1,14 & 7 & 1,00 & 6 & 18 & 5 & - \\
\hline 20 & 1,03 & 2 & 1,00 & 2 & 16 & 7 & HA \\
\hline 21 & 1,08 & 10 & 1,00 & 50 & 8 & 10 & - \\
\hline 22 & 1,07 & 5 & 1,10 & 14 & 26 & 5 & - \\
\hline 23 & 1,00 & 0 & 1,10 & 70 & 20 & 6 & - \\
\hline Médias & 1,03 & 3,52 & 1,02 & 30,4 & 14,7 & 7,5 & \\
\hline Desvio padrão & 0,04 & 3,25 & 0,04 & 18,7 & 7,5 & 1,7 & \\
\hline
\end{tabular}


obtiveram-se 5 casos normais, 2 limítrofes e $16(69,56 \%)$ anormais. A resposta da pressão arterial ao se levantar foi normal em 3 casos, limítrofe em 10 e anormal em $10(43,47 \%)$. No teste da preensão, 7 casos (30,43\%) foram anormais, 4 limítrofes e 12 normais.

\section{DISCUSSÃO}

Como pode-se constatar (Fig 1), 76,7\% dos indivíduos sadios apresentaram todos os testes normais e em $23,3 \%$ apenas um dos testes teve resultado limítrofe, enquanto que todos os diabéticos receberam escore superior a 5, e no intervalo entre os escores 2 e 4 não houve classificados.

O teste da respiração profunda mostrou-se o mais sensível (Tabela 2), apresentando resultado anormal em todos os diabéticos; com IC de $95 \%$, a sensibilidade foi $100 \%$, variando entre 82,2 e $100 \%$. Ele foi seguido pelo da manobra de Valsalva com 96,7\% de sensibilidade ( 80,9 a 99,8\%); foi $87,0 \%$ a sensibilidade para o da pressão arterial ao se levantar; 78,3\%, para o da razão. O menos sensível foi o da preensão com 47,8\%. Quando não for possível realizar os 5 testes, e em casos suspeitos, deve-se optar pelo teste da respiração profunda, seguido pelo da manobra de Valsalva.

O teste de maior especificidade é o da pressão arterial ao se levantar. Foi o único normal em todos os indivíduos sadios (Tabela 1), seguido pelo da razão e da preensão, com $96,7 \%$ de especificidade; o da respiração profunda veio a seguir, com 90,0\% e ficando com a manobra de Valsalva a menor especificidade, situada em 50,0\%. Da mesma forma, quando não for possível realizar todos os testes, e nos casos que a intenção for excluir disautonomia, a preferência recai sobre o teste da pressão arterial ao se levantar.

Embora o pequeno número de casos não tenha permitido cálculos estatísticos, pode-se constatar que o uso de medicação anti-hipertensiva não influenciou nos resultados dos testes, pois a média dos escores entre os dois grupos foi bastante próxima.

\section{CONCLUSÕES}

Os testes propostos por Ewing e cols. ${ }^{5-7}$ demonstraram ser reproduzíveis e possuidores de elevada sensibilidade e especificidade. A distribuição dos resultados em escore tem preferência, por permitir maior escalonamento da severidade. Os indivíduos classificados com escores 0 (zero) ou 1 são considerados sem evidências de disfunção autonômica; entre 2 e 4 cm, disfunção inicial e quando igual ou superior a 5, severa. Seguir a padronização proposta por Ewing demonstrou ser perfeitamente exequível em nosso meio e, quando não for possível a realização dos 5 testes, deve-se optar pelos testes da variação da frequência cardíaca durante a respiração profunda e da resposta da pressão arterial ao se levantar, por serem o mais sensível e o mais específico, respectivamente.

\section{REFERÊNCIAS}

1. Bach C, Iliana A, Eliahou HE. Autonomic nervous system disturbance in patients on chronic hemodialysis. Isr J Med Sci 1979;15:761-764.

2. Barron HV, Lesh MD. Autonomic nervous system and sudden cardiac death. J Am Coll Cardiol 1996;27:1053-1060.

3. Campese YM, Romaff MS, Levitan D et al. Mechanisms of autonomic nervous system dysfunction in uremia. Kidney Int 1981;20:246-253.

4. Consensus reached for autonomic definitions. World Neurol 1996;11:3:1-15.

5. Ewing DJ. Analysis of heart rate variability and other non-invasive tests with special reference to diabetes mellitus. In Bannister R; Mathias CJ (eds). Autonomic failure. 3 Ed. New York: Oxford, 1992: 312-333.

6. Ewing DJ, Clark BF. Diagnosis and management of diabetic autonomic neuropaty. Br Med J 1982; 285:916-918.

7. Ewing DJ, Martin CN, Yong RJ et al. The value of cardiovascular autonomic function tests: 10 years experience in diabetes. Diabetes Care 1985;8:491-498.

8. Ewing DJ, Winney R. Autonomic function in patients with chronic renal failure on intermittent hemodialysis. Nephron 1975;15:424-429.

9. Heidbreder R, Shafferhans K, Heiland A. Autonomic neuropathy in chronic renal insufficiency: comparative analysis of diabetic and nondiabetic patients. Nephron 1985;41:50-56.

10. Mailik S, Winney RJ, Ewing DJ. Chronic renal failure and cardiovascular autonomic function. Nephron 1986;43:191-195.

11. Naik RB, Mathias CJ, Wilson CA et al. Cardiovascular and autonomic reflexes in hemodialysis patients. Clin Sci 1981;60:165-170.

12. San Antonio Conference on Diabetic Neuropathy. Report and recommendations of San Antonio conference on diabetic neurophaty. Diabetes 1988;37:1000-1004. 